\title{
HIGH FREQUENCY QUANTITATIVE ULTRASOUND IMAGING OF SOLID TUMORS IN MICE
}

\author{
M.L. Oelze, W.D. O’Brien, Jr., J.F. Zachary \\ Department of Elecrtical and Computer Engineering, University of Illinois at Urbana- \\ Champaig; Department of Bioengineering, University of Illinois at Urbana-Champaign; \\ Department of Veterinary Pahtobiology, University of Illinois at Urbana-Champaign
}

\begin{abstract}
A mammary carcinoma and a sarcoma were grown in mice and imaged with ultrasound transducers operating with a center frequency of $20 \mathrm{MHz}$. Quantitative ultrasound (QUS) analysis was used to characterize the tumors using the bandwidth of 10 to $25 \mathrm{MHz}$. Initial QUS estimates of the scatterer properties (average scatterer diameter and acoustic concentration) did not reveal differences between the two kinds of tumors. Examination of the tumors using light microscopy indicated definite structural differences between the two kinds of tumors. In order to draw out the structural differences with ultrasound, a higher frequency probe (center frequency measured at $70 \mathrm{MHz}$ ) was used to interrogate the two kinds of tumors and new models were applied to the QUS analysis. QUS scatterer diameter images of the tumors were constructed using the high frequency probe. Several models for scattering were implemented to obtain estimates of scatterer properties in order to relate estimated scatterer properties to real tissue microstructure. The Anderson model for scattering from a fluid-filled sphere differentiated the two kinds of tumors but did not yield scatterer property estimates that resembled underlying structure. Using the Anderson model, the average estimated scatterer diameters were $25.5 \pm 0.14 \mu \mathrm{m}$ for the carcinoma and $57.5 \pm 2.90$ for the sarcoma. A new cell model was developed, which was based on scattering from a cell by incorporating the effects of the cytoskeleton and nucleus. The new cell model yielded estimates that appeared to reflect underlying structure more accurately but did not separate the two kinds of tumors. Using the new cell model, the average estimated scatterer diameters were $15.6 \pm 2.2 \mu \mathrm{m}$ for the carcinoma and $16.8 \pm 3.82 \mu \mathrm{m}$ for the sarcoma. The new cell model yielded estimates close to the actual nuclear diameter of the cell $(13 \mu \mathrm{m})$
\end{abstract}

Key words: tissue characterization, ultrasound backscatter 


\section{INTRODUCTION}

Cancer has become the leading cause of death in the United States of America [1]. An important factor in improving the prognosis of cancer is the early detection of cancer. Conventional ultrasound imaging can identify some types of cancerous lesions but in most cases cannot specify whether the lesion is malignant or benign.

Recent advances in a novel ultrasound imaging technique, called quantitative ultrasound imaging (QUS), have exhibited the capability to differentiate malignant tumors from benign tumors in rat and mouse models [2]. QUS imaging utilizes the frequency-dependent information that is not used in conventional ultrasound imaging [3]. The goal of QUS imaging is twofold. The first goal of QUS imaging is to distinguish between tissues that are not distinguishable through conventional ultrasound. The second goal of QUS imaging is to relate quantitative estimates (e.g. scatterer size and acoustic concentration of scatterers) to underlying microstructure.

In a recent study, three types of solid tumors in rats and mice were examined with ultrasound [4]. The tumors were a spontaneously occurring fibroadenoma, a carcinoma and a sarcoma. QUS imaging techniques were used to differentiate the three kinds of tumors at frequencies below $25 \mathrm{MHz}$. The QUS techniques were able to differentiate the benign fibroadenomas from the other two kinds of tumors. However, QUS was not able to differentiate between the carcinoma and sarcoma. Examination of optical photomicrographs of the three tumors showed distinct features of the microstructure.

In order to distinguish between the carcinoma and sarcoma, a new high frequency scanning system was used to create images. In addition, new models were developed to relate the backscatter to structures observed from optical photomicrographs of the tissues.

\section{MEASUREMENT SYSTEM}

Measurements of the carcinoma and sarcoma tumors were conducted using a high frequency transducer. The transducer was a lithium niobate transducer (Penn State). The transducer had a center frequency of $70 \mathrm{MHz}$ measured from a planar glass reflector at the focus. The diameter of the transducer was $2 \mathrm{~mm}$ with a $7-\mathrm{mm}$ focus. The analysis bandwidth used with the transducer was $30-90 \mathrm{MHz}$. The transducer was found to be highly sensitive to tissue scattering measurements.

The tumors were obtained from commercially available tumor cell lines, the 4T1 MMT carcinoma for mice and the EHS sarcoma for mice. The cells 
were cultured in medium and then injected subcutaneously into the fatpad of mice (balb/c mice for the carcinoma and C57BL/6 mice for the sarcoma). Tumors were grown to a little over a centimeter in size and then examined using QUS techniques.

\section{MODELS}

Three models were used or developed to describe the scattering from tissues. Each model assumed the Born approximation. The Born approximation is valid when there is weak scattering and no multiple scattering. The first model was the spherical Gaussian model [5], the second model was derived from the Anderson model for fluid-filled sphere [6], and the third model was constructed by considering the cytoskeleton of cells.

The spherical Gaussian model was used to describe the high frequency scattering initially because the spherical Gaussian model has been used previously to estimate scatterer properties in may tissues [3]. The spherical Gaussian model assumes that the spatial impedance distribution within the scatterers is continuously increasing over the background. The spatial variation of impedance follows a Gaussian distribution, with the largest impedance value at the center of the scatterer.

The Anderson model for fluid-filled spheres was used to describe scattering from the tissues because examination of optical photomicrographs of the carcinomas indicated that cells were the dominant source of scattering. Specifically, the nuclei of the cells were hypothesized to be a strong scatterer relative to the rest of the cell [2,7]. Therefore, the nuclei of the cells were modeled as fluid-filled spheres and the cell cytoplasm and extracellular matrix were modeled as a uniform background.

The third model incorporated not only the nucleus but also the cytoskeletal structure of the cell cytoplasm from the networks of actin filaments and microtubules. The new cell model yielded a combination of the fluid sphere model and the Gaussian model. Application of the new cell model yielded estimates of nuclear diameter.

\section{RESULTS}

QUS images of the tumors were constructed for the tumors. Figure 1 shows a QUS scatterer diameter image of a carcinoma tumor using the spherical Gaussian model. Scatterer property estimates were obtained from the carcinoma and sarcoma tumors and from pellets of the carcinoma cells. Scatterer property estimates were obtained using the three models. Relative 
chi-square values indicating the goodness-of-fit between the three models and measured data were also obtained. The average scatterer diameter and chi-square values are displayed in figure 2.

Examination of the scatterer diameter estimates indicated that the spherical Gaussian model did not yield scatterer diameter estimates that differed significantly from one tumor to the next. In addition, the chi-square value was the largest for the spherical Gaussian model indicating that the model did not fit the measured data as well as the other models.

Estimates of scatterer diameter from the Anderson model were significantly different between the carcinoma and sarcoma (average scatterer diameter of $26 \mu \mathrm{m}$ for the carcinoma versus $58 \mu \mathrm{m}$ for the sarcoma). Furthermore, the chi-square value was smaller for the Anderson model than for the spherical Gaussian model but approximately equal to the value for the new cell model. While the Anderson model distinguished between the two tumors, the diameter estimates were much larger than the actual nuclear diameters $(\sim 13 \mu \mathrm{m})$ as measured using the optical photomicrographs.

Estimates of the scatterer diameter from the new cell model yielded good fits but did not distinguish between the sarcoma and carcinoma. However, diameters estimates were very close the nuclear diameters estimated from optical photomicrographs of the tissues.
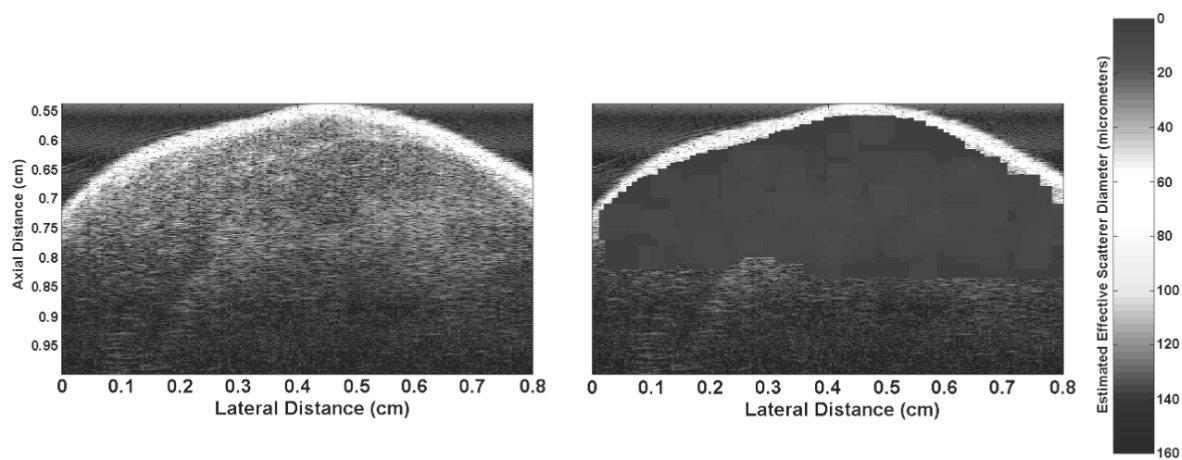

Figure 1. Ultrasound B-mode image of mouse carcinoma (left) and QUS scatterer diameter image of tumor (right) using the $70 \mathrm{MHz}$ transducer. 

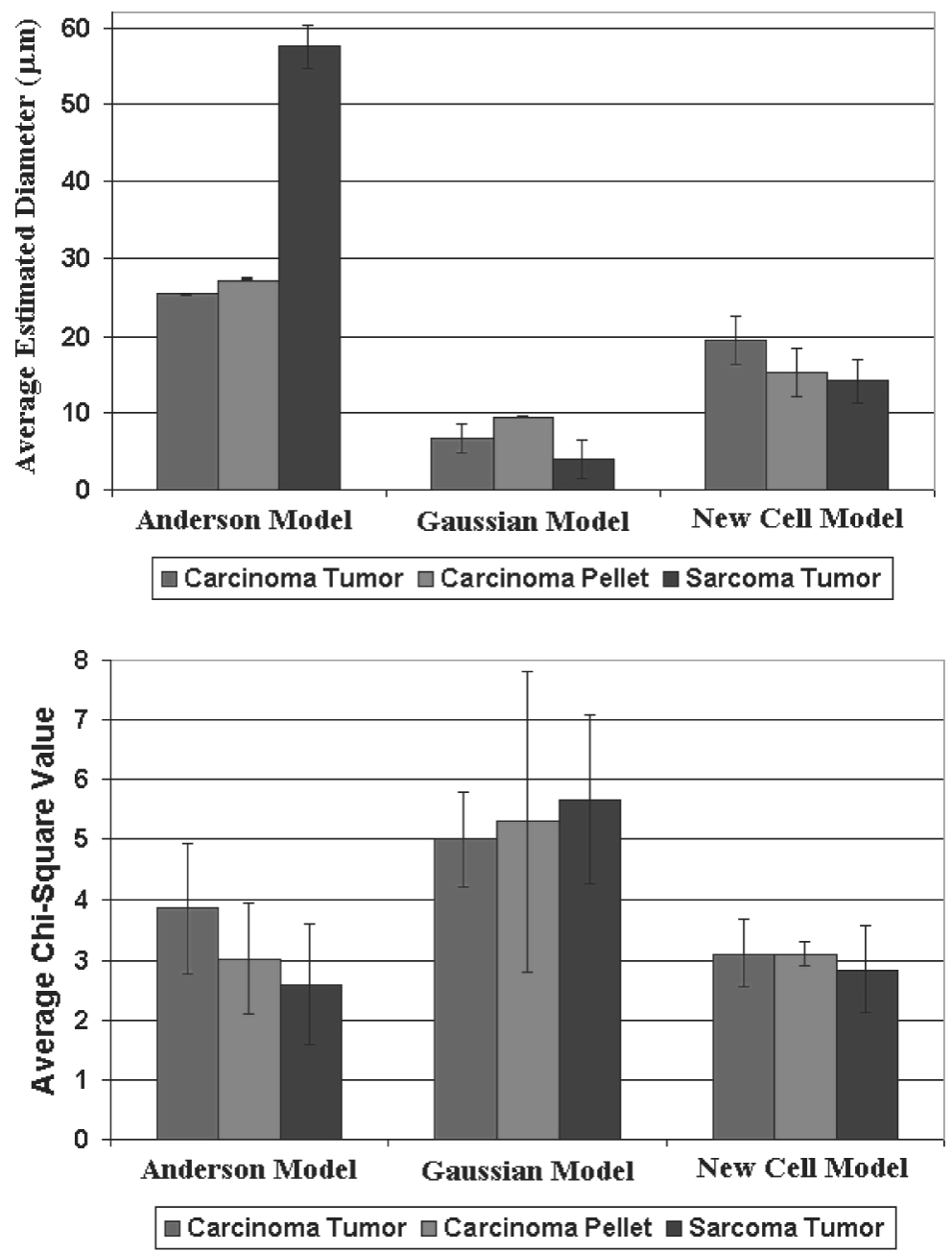

Figure 2. Average scatterer diameter estimates for the carcinoma, sarcoma and carcinoma cell pellet using the three models (left). Chi-square estimates of model fits (right). The error bars represent one standard deviation about the mean.

\section{CONCLUSIONS}

High frequency QUS imaging (30 to $90 \mathrm{MHz}$ ) was applied to two tumor models that were indistinguishable with lower frequency QUS analysis $(<25 \mathrm{MHz})$. Three new models were tested to determine if estimates of scatterer 
properties could be related to tissue microstructure and could distinguish between the two tumor models.

Use of the Anderson model for the fluid-filled sphere allowed the differentiation of the two tumor models. However, the Anderson model did not allow the correlation of scatterer property estimates to microstructure observed from optical photomicrographs of the tumor.

Use of the new cell model did not allow the differentiation of the two tumor models. However, estimates of scatterer properties derived from the model appeared to fit the underlying microstructure it was intended to model.

Differences exist between the two tumor models. These differences were exploited using the backscattered power spectrum and models to extract meaningful quantitative parameters about the microstructure. The goal of future models will be to highlight these differences and correlate with underlying structure.

\section{ACKNOWLEDGEMENTS}

The authors would like to thank James P. Blue and Rita J. Miller. This work is supported by NIH Grant F32 CA96419 to MLO.

\section{REFERENCES}

1. Jemal, A., T. Murray, E. Ward, A. Samuels, R.C. Tiwan, A. Ghafoor, E.J. Feuer, and M.J. Thun, "Cancer statistics, 2005," CA Cancer J. Clin., 55, 10-30, (2005).

2. Oelze, M.L., J.F. Zachary, and W.D. O'Brien, Jr., "Differentiation and characterization of mammary fibroadenomas and 4T1 Carcinomas using ultrasound parametric imaging," IEEE Trans. Med. Imag., 23, 764-771, (2004).

3. Oelze, M.L., J.F. Zachary, and W.D. O'Brien, Jr. "Characterization of tissue microstructure using ultrasonic backscatter: theory and technique optimization using a Gaussian form factor," J. Acoust. Soc. Am., 112, 1202-1211, (2002).

4. Oelze, M.L., W.D. O'Brien, Jr., and J.F. Zachary, "Characterization of solid tumors in mice using ultrasound backscatter over a broad range of frequencies (10 to $90 \mathrm{MHz}$ ), "4th Int. Conf. Ultra. Backscat. Micro., Arden House, New York, 2004.

5. Insana, M.F., R.F. Wagner, D.G. Brown, and T.J. Hall, "Describing small-scale structure in random media using pulse-echo ultrasound," J. Acoust. Soc. Am., 87, 179-192, (1990).

6. Anderson, V.C., "Sound scattering from a fluid sphere," J. Acoust. Soc. Am., 22, 426-431, (1950).

7. Kolios, M.C., L. Taggert, R.E. Baddour, et al., "An investigation of backscatter power spectra from cells, cell pellets and microspheres," Proc. 2003 Ultrason. Symp., 752-757, (2003). 